hep-ph/0305010

TRI-PP-03-07

UVIC-TH/05-03

\title{
Cosmological bounds on large extra dimensions from non-thermal production of Kaluza-Klein modes
}

\author{
Rouzbeh Allahverdi ${ }^{1}$, Chris Bird ${ }^{2}$, Stefan Groot Nibbelink ${ }^{2}$ \\ and Maxim Pospelov ${ }^{2,3}$ \\ 1 Theory Group, TRIUMF, 4004 Wesbrook Mall, \\ Vancouver, B.C., V6T 2A3, Canada. \\ 2 Department of Physics and Astronomy, University of Victoria, \\ Victoria, B.C., V8P 1A1, Canada. \\ 3 Centre for Theoretical Physics, University of Sussex, \\ Brighton BN1 9QJ, U.K.
}

\begin{abstract}
The existing cosmological constraints on theories with large extra dimensions rely on the thermal production of the Kaluza-Klein modes of gravitons and radions in the early Universe. Successful inflation and reheating, as well as baryogenesis, typically requires the existence of a $\mathrm{TeV}$-scale field in the bulk, most notably the inflaton. The non-thermal production of KK modes with masses of order $100 \mathrm{GeV}$ accompanying the inflaton decay sets the lower bounds on the fundamental scale $M_{*}$. For a $1 \mathrm{TeV}$ inflaton, the late decay of these modes distort the successful predictions of Big Bang Nucleosynthesis unless $M_{*}>35,13,7,5$ and $3 \mathrm{TeV}$ for $2,3,4,5$ and 6 extra dimensions, respectively. This improves the existing bounds from cosmology on $M_{*}$ for 4,5 and 6 extra dimensions. Even more stringent bounds are derived for a heavier inflaton.
\end{abstract}




\section{Introduction}

The original motivation for theories with large extra spatial dimensions was the explanation of the hierarchy between the electroweak scale and the Planck mass. In this set up our four-dimensional world is assumed to be a flat hyper surface, called the Standard Model (SM) brane, which is embedded in a higher dimensional space-time, known as the bulk. The weakness of gravity in $3+1$ dimensions is ascribed to the dilution of the gravitational interaction by the large volume of the extra dimensions transparent for gravity, but inaccessible to SM matter and gauge fields. The hierarchy problem can be solved by assuming that the fundamental scale $M_{*}$ in the $4+d$ dimensional bulk is of the order of a TeV [1, 2, 3]. However, this requires the extra dimensions to be large. More quantitative, let $R$ set the common scale of the $d$ extra compact dimensions such that their volume is $(2 \pi R)^{d}$. Then the effective four-dimensional Planck mass becomes

$$
M_{\mathrm{p}}^{2}=M_{*}^{2+d} R^{d} .
$$

This dilution of gravity results in modifications of Newton's law at distances smaller than $R$. Take $M_{*}=1 \mathrm{TeV}$ for example, then for one extra dimension, $R$ will be of order of the size of solar system, which is clearly ruled out. However, already for $d=2$ one finds a value of $R \simeq 0.2 \mathrm{~mm}$, which is in reach of current experimental searches for deviations from Newtonian gravity [4].

From an effective four dimensional perspective, the distinctive feature of large extra dimension models is the appearance of a tower of massive graviton states, known as Kaluza-Klein (KK) graviton modes. The perturbations of components of the $4+d$ dimensional metric tensor behave as a rank 2-tensor (the graviton), vectors (the graviphotons), and scalars (gravi-scalars) respectively. The diagonal part of the gravi-scalar matrix contains the $d$ so-called radions, which couple to the trace of the four dimensional energy momentum tensor. (For details see Ref. [5.) The energy of these modes is given by $E^{2}=k^{2}+\sum_{i} n_{i}^{2} R^{-2}$, where $k$ represents the three-dimensional momentum, and $n_{i} R^{-1}$ the integral internal momenta. ${ }^{1}$ Collectively, the square sum of these internal momenta looks like a mass squared, denoted by $m_{K K}^{2}$.

The coupling of each KK mode to matter is small, as it is proportional to $1 / M_{\mathrm{p}}$. Moreover, the gravi-photon has no coupling to the matter fields at the tree-level, and

\footnotetext{
${ }^{1}$ This spectrum applies to a square torus with all radii equal to $R$. In general there may exist other shape parameters that would change the precise structure of the KK towers [6]. However since we are only interested in generic effects associated with the radion and gravitons, these effects only give subleading corrections. Neither we consider more complicated models in which the KK spectrum does not depend on the size of the extra dimensions and very heavy KK modes can emerge from large extra dimensions [7].
} 
hence can be ignored in the subsequent discussion. The coupling of a radion to fermions is proportional to the fermion mass $m_{f}$; its coupling to massless gauge bosons vanishes at the tree-level.

The coupling, and therefore the emission probability, of a particular KK mode is very small due to the large size of extra dimensions as compared to the electroweak scale. However, due to the small energy level spacing between the KK modes, the multiplicity of kinematically allowed KK modes grows rapidly with energy. The resulting probability of emission of KK modes scales like $(E R)^{d+2} E^{2} M_{\mathrm{p}}^{-2} \sim\left(E / M_{*}\right)^{d+2}$. This has an important impact on particle physics through the production of on-shell and exchange of off-shell KK modes in various processes. Collider experiment constraints on the fundamental scale $M_{*}$ are comparable to $1 \mathrm{TeV}$ scale 8 . Much more stringent bounds can be derived from cosmology and astrophysics, as we review in section 2 .

All cosmological bounds on the scale of extra dimensions derived in the literature rely on thermal production of the KK modes from the scattering of SM particles. It is usually assumed that the temperature is at its lowest possible value consistent with Big Bang Nucleosynthesis (BBN) [9], i.e. $\gtrsim 0.7 \mathrm{MeV}$ [10]. However, it is extremely problematic to start the history of the Universe from a thermal state with such a low temperature without having a pre-thermal history. Standard cosmological problems such as flatness, isotropy, etc., prompt the introduction of appropriate inflationary models in the context of extra dimensions [11, 12, 13, 14]. Inflation in models with large extra dimensions can be quite different from well-studied inflationary scenarios in four dimensional theories. The inflaton has to be a bulk field, since the possibility of a brane inflaton is not really viable [12: for $M_{*} \sim \mathcal{O}(\mathrm{TeV})$ this requires very unnatural initial conditions, as well as an inflaton which is too light to generate density perturbations of the correct amplitude. The size of the extra dimensions needs to be stabilized before the time of BBN, otherwise the observationally tightly constrained abundances of light elements [15] will be distorted. We assume that extra dimensions were somehow stabilized before, or during inflation [16], so that for the last e-foldings of inflation, relevant for the observable density perturbations, only three ordinary dimensions are still expanding [14. The requirement of reheating before $\mathrm{BBN}$ imposes bounds on the width and the mass of the inflaton field, with the latter being on the order or larger than the electroweak scale $v_{E W}$. In addition, baryogenesis in models with large extra dimensions [17] also requires the existence of fields masses larger than $v_{E W}$ [18].

Therefore, in all realistic scenarios studied to date, the Universe had to pass through a stage of entropy production in which the characteristic energy/momentum transfers were at least of the order of the electroweak scale. For simplicity we refer to the field $\phi$ which is responsible for this stage as the "inflaton" in this work. Since gravity is the universal force which couples to all energy sources, the decay of the inflaton is necessarily 
accompanied by non-thermal emission of KK gravitons with masses comparable to $m_{\phi}$. The resulting KK excitations may survive for several years, and decay when all light elements have already been formed. Their decay products may distort the abundance of light elements predicted from BBN. Consequently, as we will quantify in this paper, these non-thermal processes can strengthen the cosmological bounds on large extra dimensions, especially if $d \gtrsim 3$.

This paper is organized as follows. Section 2 reviews previously obtained bounds on the scale of the large extra dimensions using astrophysical and cosmological probes. In section 3 we first argue that non-thermal production of KK modes is inevitable in models with large extra dimensions because of the need of bulk inflation. After that, we identify non-thermal processes of KK mode production, like the decay of a heavy inflaton, and investigate their potential to constrain $M_{*}$ in various extra dimensions. Our main bounds can be found in Table 2. Section 4 summarizes our conclusions. The calculations of the emission of KK radions and gravitons in the decay of the inflaton are summarized in Appendix A

\section{Cosmological bounds on the scale of extra dimen- sions via thermal production of $\mathrm{KK}$ modes}

This section gives a short overview of the various bounds on $M_{*}$, as a function of the number of extra dimensions $d$, that have been obtained from various cosmological and astrophysical considerations. We also briefly indicate what kind of physics has been employed to obtain these bounds.

After reheating $T<T_{\mathrm{R}}$, the Universe should be described by a thermal bath, which is radiation-dominated at least during $\mathrm{BBN}, T_{\mathrm{R}} \gtrsim 0.7 \mathrm{MeV}$ [10; otherwise the wellmeasured abundances of the light elements become distorted. However, since the massive KK modes behave as non-relativistic matter, their existence may be problematic. First of all, above the so-called normalcy temperature $T_{\mathrm{N}}^{d+1} \sim M_{*}^{d+2} / M_{\mathrm{p}}$ the $\mathrm{KK}$ modes dominate, hence one requires that $T_{\mathrm{R}}<T_{\mathrm{N}}$ [1]. In addition, KK modes redshift more slowly than radiation, and therefore will become dominant eventually, provided that their lifetime is sufficiently long. This results in a tight bound on $M_{*}$ from the overclosure limit. For example, for $d=2$ and $T_{\mathrm{R}} \gtrsim 3 \mathrm{MeV}$, one finds $M_{*} \gtrsim 40 \mathrm{TeV}$ [19]. This bound on $M_{*}$ can be improved by considering the diffuse photon background, produced in the decay of a $\mathrm{KK}$ graviton into a pair of photons in the $\mathrm{MeV}$ range [19]. This diffuse gamma background has been measured by the EGRET [20] and COMPTEL 21] experiments, and from this the bound $M_{*} \gtrsim 110 \mathrm{TeV}$ for $d=2$ follows [19]. 


\begin{tabular}{|c||c|c|c|c|c|}
\hline Bounds from & $d=2$ & $d=3$ & $d=4$ & $d=5$ & $d=6$ \\
\hline \hline cosmology & $167 \mathrm{TeV}$ & $21.7 \mathrm{TeV}$ & $4.75 \mathrm{TeV}$ & $1.55 \mathrm{TeV}$ & $<1 \mathrm{TeV}^{\dagger}$ \\
astrophysics & $3930 \mathrm{TeV}$ & $146 \mathrm{TeV}$ & $16.1 \mathrm{TeV}$ & $3.4 \mathrm{TeV}$ & $1 \mathrm{TeV}$ \\
\hline
\end{tabular}

Table 1: The tightest current cosmological [25] and astrophysical [27] bounds on the Planck scale in $4+d$ dimensions $M_{*}$, for $2 \leq d \leq 6$. Note that with our defintion of the fundamental scale, Eq. (1), $M_{*}$ coincides with $M$ in Ref. [25], while related to $M$ in Ref. 27] through the relation $M_{*}=(4 \pi)^{1 / d+2} M .{ }^{\dagger}$ A cosmological bound for $d=6$ has not been given in [25]; but it is certainly less than $1 \mathrm{TeV}$.

Even more stringent bounds can be obtained from KK modes, which are thermally produced during reheating. In this epoch the Universe is matter-dominated and an instantaneous thermal bath exists [22, with maximum temperature $T_{\max }$ 23, 24], which carries a small, but growing, fraction of the total energy density. Due to the continuous injection of entropy from inflaton decay, $T$ decreases more slowly than if radiation were dominant. As a consequence, the number density of produced particles is redshifted more rapidly as a function of temperature, i.e. $\propto T^{8}$, instead of $\propto T^{3}$ for radiation [22]. The KK modes with masses $T_{\mathrm{R}}<m_{K K}<T_{\max }$ are produced with only a power-law suppression during reheating, rather than an exponential suppression in a radiationdominated Universe. This has been used in Ref. [25] to tighten the bound on $M_{*}$. In fact, with the lowest BBN allowed reheat temperature, $T_{\mathrm{R}} \approx 0.7 \mathrm{MeV}$ and $T_{\max }=1$ $\mathrm{GeV}$, a bound of $M_{*} \gtrsim 167(21.7) \mathrm{TeV}$ has been obtained for $d=2$ (3).

The KK modes production in hot and dense astrophysical objects also leads to very tight bounds on $M_{*}$ [26, 27]. For 2 extra dimensions one finds in this way $M_{*} \gtrsim 3900$ $\mathrm{TeV}$ [27.

Both the cosmological and astrophysical bounds rely on the thermal production of KK modes at temperatures in the range of 1 to $100 \mathrm{MeV}$. The probability of a KK mode production in a single fusion process scales as $\left(T / M_{*}\right)^{d+2}$; hence for larger $d$ the bounds derived from such processes do not give very stringent constraints. The strongest cosmological and astrophysical bounds, which are currently available, have been listed in Table 1 

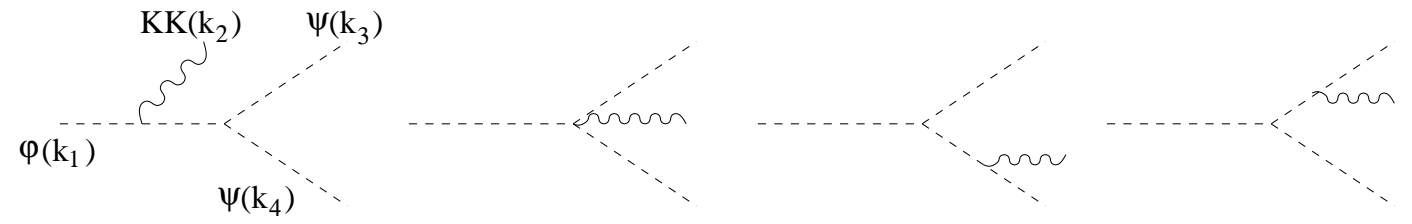

Figure 1: The associated emission of KK modes in the decay of the inflaton into a pair of the scalars $\psi$ from the incoming leg, the vertex, and the outgoing legs.

\section{Non-thermal production of KK modes in inflaton decay}

As recalled in the introduction, the existence of a bulk inflaton is a fairly generic feature of viable inflationary scenarios in large extra dimensions. This inflaton has to produce the SM fields, which live on the brane, in order to reheat the Universe. This can either be a direct process, or the inflaton first decays to other bulk or brane fields, which in turn decay to the SM particles. In either case, the reheat temperature $T_{\mathrm{R}}$ must be sufficiently low to avoid thermal overproduction of KK modes, as mentioned in section 2. A higher $T_{\mathrm{R}}$ would require a larger $M_{*}$, or late entropy release in order to dilute the KK modes in excess. Note that the energy density of a brane field is in general too small to accomplish this. In conclusion, the situation is that the decay of a bulk field will typically be responsible for the final stage of reheating, which yields $T_{\mathrm{R}} \simeq 1 \mathrm{MeV}$.

The decay rate of the inflaton depends on its coupling to the SM fields. The decay to the Higgs, to fermions, and to gauge fields corresponds to coupling through a dimension $D=3,4$, and 5 operator, respectively. After taking into account the volume suppression factor, the resulting decay widths scale like

$$
\Gamma_{D} \sim \frac{M_{*}^{3}}{M_{P}^{2}}\left(\frac{m_{\phi}}{M_{*}}\right)^{2 D-7} .
$$

(The minimum $D=3$ corresponds to a $\phi \mathrm{H}^{\dagger} \mathrm{H}$ operator, in which case Eq. (2) is applicable only if $m_{\phi}>2 m_{H}$.) Recall that for successful BBN, the total decay width $\Gamma_{t o t}$ of the inflaton has to satisfy $\Gamma_{\text {tot }} \sim T_{\mathrm{R}}^{2} / M_{\mathrm{P}}$, with the reheat temperature $T_{\mathrm{R}} \gtrsim 0.7 \mathrm{MeV}$. With $M_{*}$ not hierarchically larger than $1 \mathrm{TeV}$, this implies that $m_{\phi}$ must be of the order of 1 $\mathrm{TeV}$, as well. Note that this is, in fact, a generic conclusion for the decay of any bulk field into fields localized on the brane.

The universal character of the gravitational interaction ensures that any decay of the inflaton $\phi \rightarrow \psi_{i} \psi_{j}$ to the SM fields $\psi_{i}$ is accompanied by KK mode emitting processes 
$\phi \rightarrow \psi_{i} \psi_{j}+K K$ with $m_{K K} \lesssim m_{\phi}{ }^{2}$ These KK modes may carry a considerable fraction of the energy of the decaying inflaton. Let us concentrate on the inflaton decay into the Higgs scalars. As we shall see below, the coupling constant of the inflaton to matter does not enter in the expression for the total energy emitted to KK modes, and hence our analysis is rather insensitive to the nature of the SM final states, and their coupling to the inflaton. The diagrams describing the emission of KK radions and gravitons in the decay of the inflaton to two scalars are depicted in Fig. I. (KK modes with $\mathcal{O}(100 \mathrm{GeV}$ ) masses are also produced during reheating, provided that $T_{\max }$ is sufficiently large. However, compared to those produced in inflaton decay, their abundances are negligible, as they are suppressed by large powers of $T_{\mathrm{R}} / m_{K K}$.)

For the KK modes decaying after recombination, i.e. $\tau_{K K}>10^{12} \mathrm{sec}$, the gamma ray background provides the strongest bound on the total energy $\sum n_{K K} \cdot m_{K K} / s$ released in KK modes [29, 30]. (Here $\sum$ denotes the sum over all KK modes which are kinematically allowed.) For shorter lifetimes, $\tau_{K K}<10^{12} \mathrm{sec}$, the constraints from dissociation of primordial light elements by KK mode decay products are stronger [29, 31]. The most stringent $\mathrm{BBN}$ bound arises for $10^{8} \mathrm{sec} \lesssim \tau_{K K} \lesssim 10^{12} \mathrm{sec}$, and reads [31]

$$
\frac{\sum n_{K K} \cdot m_{K K}}{s \cdot 1 \mathrm{GeV}} \simeq 2 \cdot 10^{-12}
$$

The energy emitted into KK modes with even shorter lifetimes, $10^{6}$ sec $\lesssim \tau_{K K} \lesssim 10^{8}$ sec, is bounded by the BBN predictions at the level of $2 \cdot 10^{-9}$ [31. Such lifetimes correspond to masses of KK modes comparable to the electroweak scale. Indeed, KK gravitons with a mass larger than the electroweak scale decay to all SM particles with a decay lifetime 32]

$$
\tau_{\mathrm{gr}} \simeq 5 \times 10^{4} \mathrm{sec}\left(\frac{1 \mathrm{TeV}}{m_{K K}}\right)^{3},
$$

while the lifetime of the radions, capable to decay into $\mathrm{HH}, W^{+} W^{-}, Z Z$, is given by 32

$$
\tau_{\text {rad }} \simeq 2(d+2) \times 10^{5} \sec \left(\frac{1 \mathrm{TeV}}{m_{K K}}\right)^{3}
$$

The lifetime of massive radions is substantially longer than that of massive gravitons, because the latter has a lesser number of efficient decay channels. The $\tau_{i}$ dependence on the KK mass $m_{K K}$ is plotted in Fig. 2. Assuming that the decaying inflaton is heavier

\footnotetext{
${ }^{2}$ In general, particles with a mass $\lesssim m_{\phi}$ are produced in inflaton decay in higher orders of perturbation theory whenever they can be produced via thermalized inflaton decay products [28].
} 


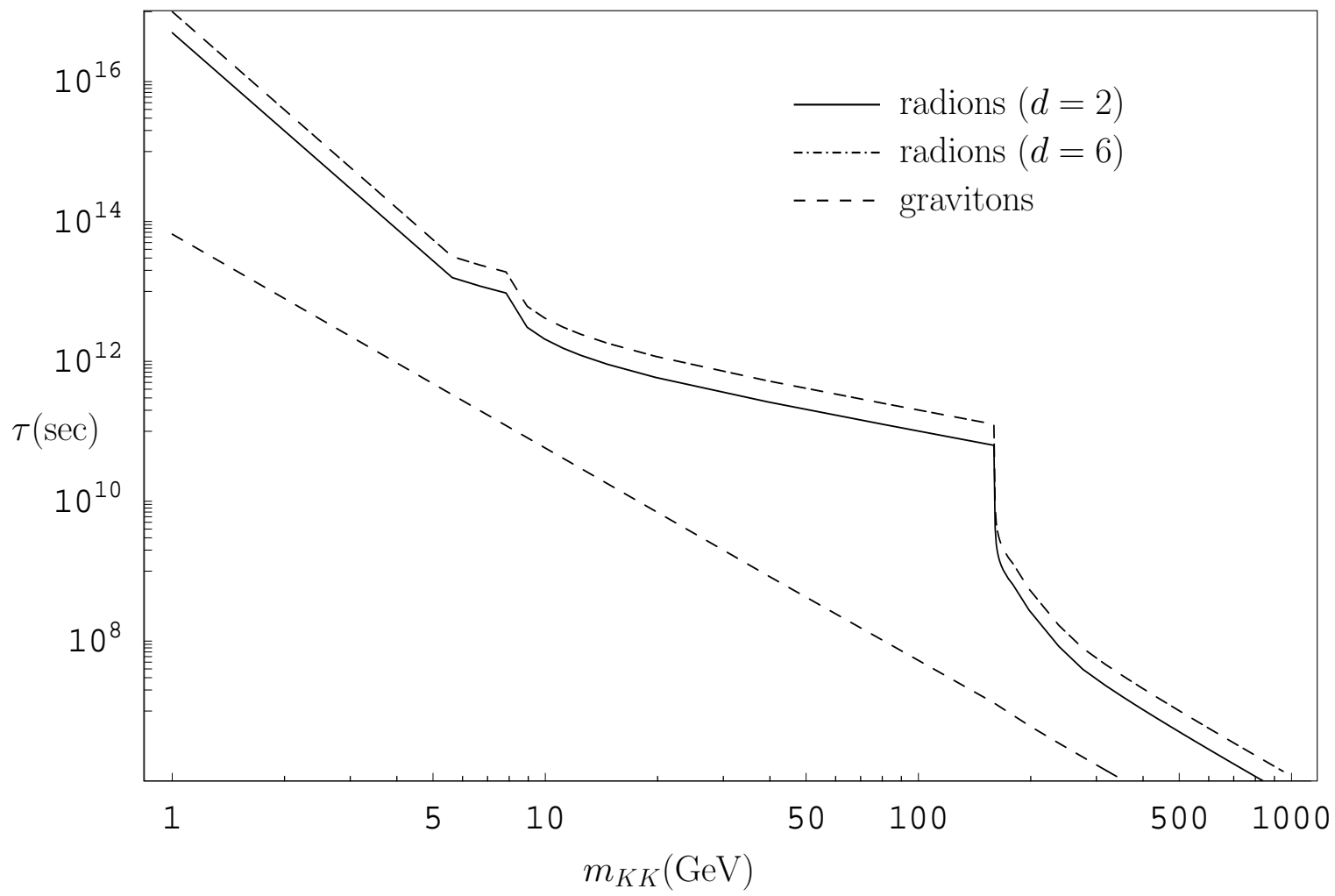

Figure 2: The lifetime of the KK gravitons as a function of their mass is indicated by the dashed line. The lifetime dependence of the KK radions on the number of extra dimensions is shown by the solid line for $d=2$ and the dashed-dotted line for $d=6$. In addition, the radion lifetime is very sensitive to $m_{K K}$ near the $W W$ threshold.

than all massive KK excitations,

$$
\begin{array}{ll}
m_{\max }^{\mathrm{gr}} \simeq 80 \mathrm{GeV} ; & m_{\max }^{\mathrm{rad}} \simeq 200,210,225, \quad 240,250 \mathrm{GeV}, \\
\text { for } d=2, \quad 3, \quad 4, \quad 5, \quad 6,
\end{array}
$$

are the maximum masses of the KK gravitons and radions, respectively, which give the lifetime of $10^{8}$ sec and allow to use the tight bound (3).

We now compute the energy density carried by radions and gravitons with $m_{K K} \leq$ $m_{\max }$ based on decay width calculations presented in Appendix A. The ratio of the number density of emitted single KK mode over the number density of the inflaton $n_{\phi}$, 


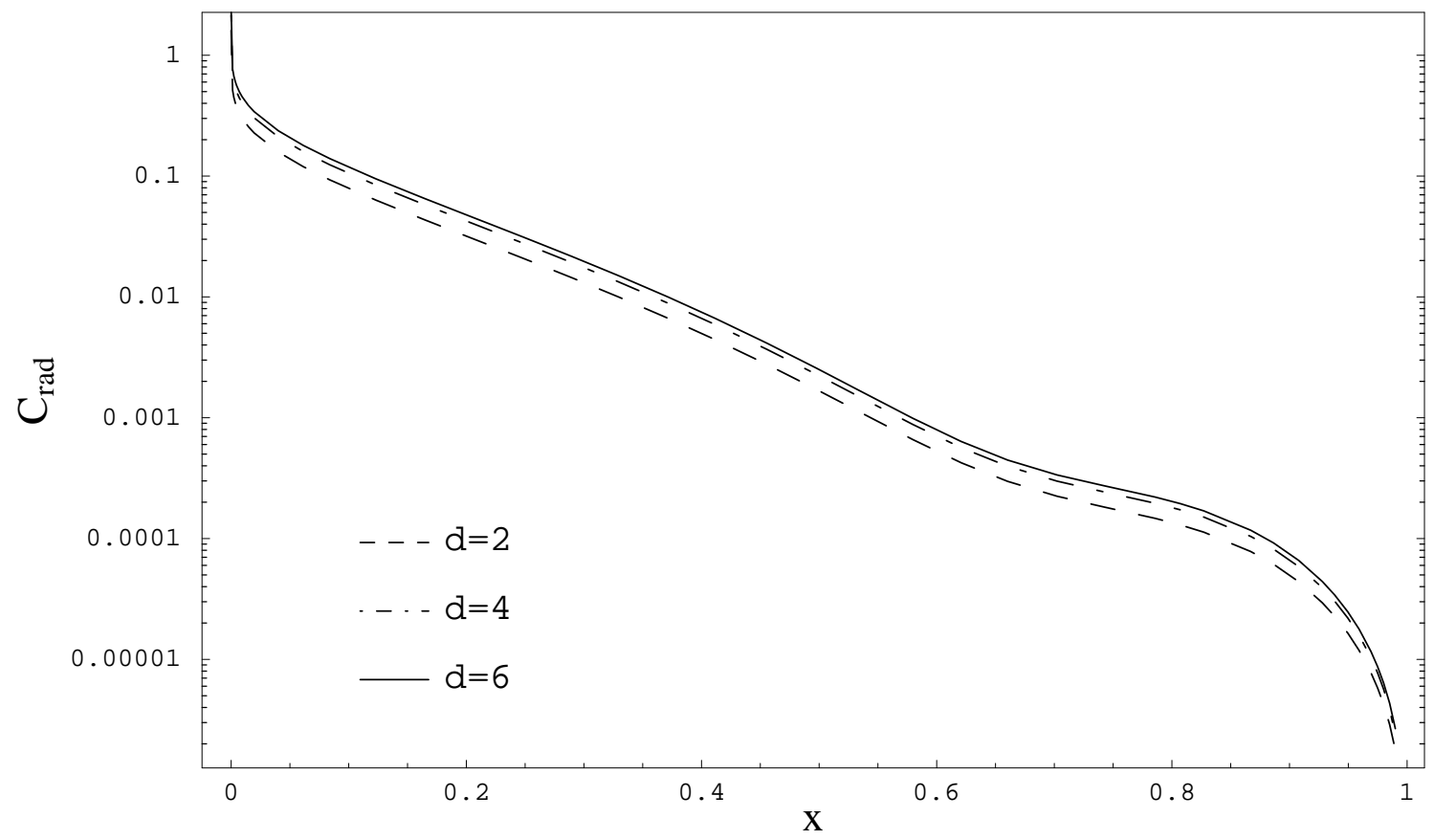

Figure 3: The dependence of the factor $C_{\mathrm{rad}}(x)$, defined in (17), on the ratio $x=m_{K K}^{\mathrm{rad}} / m_{\phi}$ of the radion over the inflaton mass is plotted for the number of extra dimensions $d=3,4$, and 6 .

can be expressed in terms of a function $C_{i}(x)$ as

$$
\frac{n_{K K}^{i}}{n_{\phi}}=C_{i}(x) \frac{m_{\phi}^{2}}{M_{\mathrm{P}}^{2}}, \quad x=\frac{m_{K K}^{i}}{m_{\phi}}, \quad i=\mathrm{gr}, \mathrm{rad} .
$$

The functions $C_{i}(x)$ depend on the dimension $d$, and are different for radions and gravitons, and have been computed in Appendix A In particular, their asymptotic expressions have been given in (17) and (19) of this Appendix. In Fig. 3] we have plotted $C_{\text {rad }}(x)$ for $d=2,4$, and 6 .

By combining the expressions for the number densities (17) with the entropy released by inflaton decay

$$
\frac{n_{\phi}}{s} \simeq \frac{3 T}{m_{\phi}}
$$

we arrive at the expression for the energy density of KK modes weighted with entropy 


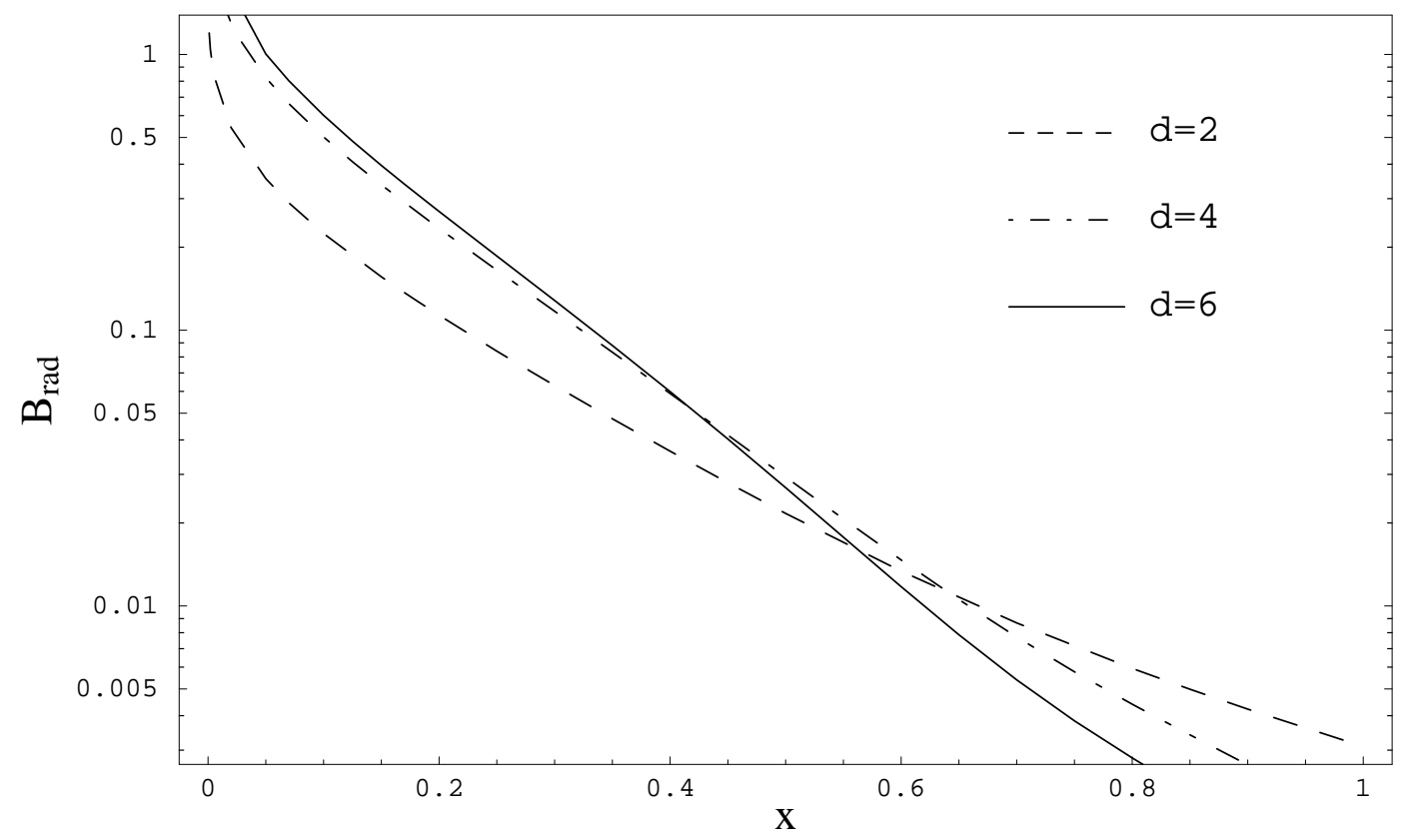

Figure 4: The dependence of $B_{\text {rad }}(x)$, given in (91), on the ratio $x=m_{\max }^{\mathrm{rad}} / m_{\phi}$ is presented for the number of extra dimensions $d=2,4$, and 6 .

factor

$$
\frac{1}{s} \sum m_{K K}^{i} n_{K K}^{i} \simeq 3 B_{i}(x) \frac{T m_{\phi}}{M_{*}}\left(\frac{m_{\max }^{i}}{M_{*}}\right)^{d+1}, \quad x=\frac{m_{\max }^{i}}{m_{\phi}} .
$$

Here the functions $B_{i}(x)$ have been obtained by summing $\sum m_{K K}^{i} C\left(m_{K K}^{i} / m_{\phi}\right)$ over all KK modes with a mass smaller than the maximal KK mass $m_{\max }^{i}$. Since the spacing of modes is $1 / R$, we may replace this sum by an integral over a sphere of radius $R m_{\text {max }}^{i}$, which, after some change of variables, becomes

$$
B_{i}(x)=\frac{S_{d}}{x^{d+1}} \int_{0}^{x} d y C_{i}(y) y^{d}, \quad x=\frac{m_{\max }^{i}}{m_{\phi}}
$$

where $S_{d}=2 \pi^{d / 2} / \Gamma(d / 2)$ is the surface area of the $d$-dimensional unit sphere. For the radion and $d=2,4$, and 6 , we have plotted the function $B_{\text {rad }}(x)$, see Fig. 4 , In the limit $x=m_{\max }^{i} / m_{\phi} \ll 1, B_{i}(x)$ and $C_{i}(x)$ are related by $B_{i}(x) \sim C_{i}(x) S_{d} /(d+1)$. The expressions in (9) remain approximately constant in the subsequent thermal evolution, since the entropy and KK number densities redshift in the same manner. 


\begin{tabular}{|c||c|c|c|c|c|}
\hline$m_{\phi}$ & $d=2$ & $d=3$ & $d=4$ & $d=5$ & $d=6$ \\
\hline \hline $1 \mathrm{TeV}$ & $35 \mathrm{TeV}$ & $13 \mathrm{TeV}$ & $7.1 \mathrm{TeV}$ & $4.5 \mathrm{TeV}$ & $2.8 \mathrm{TeV}$ \\
$2 \mathrm{TeV}$ & $47 \mathrm{TeV}$ & $17 \mathrm{TeV}$ & $9.1 \mathrm{TeV}$ & $5.7 \mathrm{TeV}$ & $3.4 \mathrm{TeV}$ \\
$M_{*}$ & $220 \mathrm{TeV}$ & $42 \mathrm{TeV}$ & $15 \mathrm{TeV}$ & $7.9 \mathrm{TeV}$ & $4.0 \mathrm{TeV}$ \\
\hline
\end{tabular}

Table 2: The cosmological bounds on the Planck scale in $4+d$ dimensions $M_{*}$, for $2 \leq d \leq 6$ and inflaton masses $m_{\phi}=1,2 \mathrm{TeV}$ and $M_{*}$, are obtained by requiring that late decay of KK excitations do not distort the BBN predictions. These bounds can be compared with the previous bounds collected in Table 1

From now on we assume, that $T \simeq T_{\mathrm{R}} \approx 0.7 \mathrm{MeV}$, since higher reheat temperatures only lead to stronger bounds. In the asymptotic regime of $x=m_{\max } / m_{\phi} \ll 1$ the average non-relativistic energy density carried by the sum of KK radions and gravitons can be written as

$$
\frac{\sum m_{K K} n_{K K}}{s} \simeq \frac{2 S_{d}}{(d+1) \pi} \frac{T_{\mathrm{R}} m_{\phi}}{M_{*}}\left[\frac{d}{d+2}\left(\frac{m_{\max }^{\mathrm{rad}}}{M_{*}}\right)^{d+1}+2\left(\frac{m_{\max }^{\mathrm{gr}}}{M_{*}}\right)^{d+1}\right] \ln \left(\frac{m_{\phi}}{m_{\max }}\right),
$$

where we have used the approximation $\ln m_{\max }^{\mathrm{rad}} \simeq \ln m_{\text {max }}^{\mathrm{gr}}$. Imposing the constraint (3i) on (11), we arrive at a set of bounds on $M_{*}$, which are summarized in the Table 2. The strength of these limits is mainly due to $200 \mathrm{GeV}$ radions. The contribution of the gravitons is small, and does not exceed $15 \%$ of the total $\sum m_{K K} n_{K K}$, because of a factor of 3 difference in the maximum values of $m_{K K}$ for radions and gravitons, given in (6). Below (3) we noted that for lifetimes of $10^{6} \mathrm{sec} \lesssim \tau_{K K} \lesssim 10^{8} \mathrm{sec}$ of KK modes, the BBN bound is weakened to $2 \cdot 10^{-9}$ [31]. However, using these numbers instead, does not affect the limit on $M_{*}$ quoted in Table 2 much for $m_{\phi}=1 \mathrm{TeV}$. For heavier inflatons the limits would be strengthened. For example, for $d=6$ and $m_{\phi}=2 \mathrm{TeV}$, the bound on $M_{*}$ is at the $4 \mathrm{TeV}$ level.

It is important to note that in the approximation $m_{\max } \ll m_{\phi}$ the first diagram in Fig. [1 dominates and contributes to Eq. (111). Therefore, in this approximation, the reheating to other particles would produce the same answer. We checked that this is indeed the case for the reheating into a pair of fermions. Thus, our bounds are practically insensitive to the nature of the inflaton-matter coupling.

Associated inflaton decay into KK modes calculated in this section is not the only source of non-thermal production of radions and gravitons. KK modes will also be produced during the thermalization process of the inflaton decay products. In this process 
energetic inflaton decay products scatter among themselves and with the particles in the thermal bath, which have energies of the order of the reheat temperature $T_{\mathrm{R}}$ [24]. The scattering of energetic particles with energies $E \sim m_{\phi} / 2$ would lead to a production of KK modes with masses up to $m_{K K} \lesssim m_{\phi}$. An estimate of $s^{-1} \sum m_{K K} n_{K K}$ produced this way shows that it is subdominant to (91). The main reason for this is, that the scattering of the inflaton decay products is suppressed by the square of rather small number density of energetic particles. This leads to additional suppression by a square of the small parameter $T_{\mathrm{R}} / m_{\phi}$. The scattering of the hard particles on the particles from the thermal bath may produce KK modes with masses $m_{K K} \lesssim\left(T m_{\phi}\right)^{1 / 2}$. This domain of KK masses is rather small to be important for the production of the KK modes in $d>3$ models. Estimates of the energy densities of the KK modes producing the diffuse photon background for $d=2,3$ models show that the resulting level of sensitivity to $M_{*}$ is lower than previously found in Refs. [19, 25]. It is interesting to note in passing that the largest production of KK modes during thermalization is expected from the thermalization of neutrinos because they interact via the weak force with the thermal bath and thus exist much longer in this environment than the rest of the particles.

\section{Conclusions}

We have presented a derivation of cosmological bounds on the fundamental scale of quantum gravity $M_{*}$ in large extra dimension models. These bounds are obtained by utilizing the non-thermal production of massive KK gravitons and radions from the decay of a heavy inflaton field. Such a heavy field seems to be a generic feature of models with large extra dimensions: Inflationary resolutions of standard cosmological problems together with successful reheating require, that the inflaton mass is larger than the electroweak scale. Moreover, it seems to be impossible to generate the baryon asymmetry of the Universe without invoking some physics beyond the electroweak scale. Our limits on $M_{*}$ arise because the subsequent decay of those KK modes after the BBN epoch may distort the abundances of light elements, which are tightly constrained by observations.

The production of KK modes during inflaton decay cannot be computed in a fully model-independent way. However, the (potentially) most uncertain parameter, the inflaton coupling to the matter, dropped out of the calculation of the total energy density of KK modes. Also the reheating into fermions or gauge bosons, instead of only scalars as we considered, will not drastically change the conclusions from the calculation. This possibly leads to an order unity change in numerical coefficients, but the dependence on the main parameters $m_{\max }, m_{\phi}$ and $M_{*}$ remains the same as in (11). 
Our limits on the fundamental scale $M_{*}$ have been summarized in Table 2 For any number of dimensions the resulting limits are stronger than $2 \mathrm{TeV}$. Hence, they are clearly superior to existing high-energy physics bounds, and very competitive with any possible level of sensitivity to $M_{*}$ of future collider experiments of this decade. For a TeV scale inflaton the cosmological bounds found in the literature [19, 25] are stronger than ours for 2 or 3 extra dimensions. However, our limits can become more stringent for a heavier inflaton. Indeed, the saturated bounds in Table 2, obtained for $m_{\phi}=M_{*}$, are certainly tighter than the existing ones. For $m_{\phi} \geq 1 \mathrm{TeV}$, our limits are better than the current strongest one for $d=4$ [25], and are clearly an improvement for $d=5$ and 6. The most stringent astrophysical bounds which currently exist [27] are much stronger than our bounds for $d=2,3$, and are better for $d=4$. However, our bounds are again superior for $d=5,6$.

The reason that our bounds are particularly powerful for large $d$ is the following. The existing cosmological bounds rely on thermal production of KK modes, which have much smaller $m_{K K}$. Therefore, even though their abundances are enhanced by a large time of emission, for sufficiently large $d$ the phase space factor takes over, and results in a larger abundance for non-thermally produced KK modes from inflaton decay.

\section{Acknowledgements}

R.A. and S.G.N. wish to thank kind hospitality by the Department of Physics and Astronomy at the University of Victoria, and the Theory group of TRIUMF. M.P. would like to thank I. Mocioiu for useful discussions at the initial stage of this project.

This work has been supported by the NSERC of Canada. In addition, S.G.N. acknowledges the National Fellowship support of CITA, and M.P. the support of PPARC of the UK.

\section{A Emission of KK modes}

In section 3 we discuss how the inflaton decay to KK gravitons and radions can be used to put bounds on the fundamental $4+d$ dimensional Planck scale $M_{*}$. This appendix is devoted to the calculation of an estimate of the ratio of the number density of produced KK modes $n_{K K}^{i}$ to the number density of the inflaton $n_{\phi}$ on which these bounds crucially rely. This ratio is equal to the three body decay width containing a single $\mathrm{KK}$ mode $\Gamma_{3 i}$ 
over the total decay width $\Gamma_{\text {tot }}$ of the inflation:

$$
C_{i}(x) \frac{m_{\phi}^{2}}{M_{\mathrm{P}}^{2}} \equiv \frac{n_{K K}^{i}}{n_{\phi}}=\frac{\Gamma_{3 i}}{\Gamma_{t o t}} \simeq \frac{\Gamma_{3 i}}{\Gamma_{2}}, \quad x=\frac{m_{K K}^{i}}{m_{\phi}}, \quad i=\mathrm{rad}, \mathrm{gr} .
$$

Here we have assumed that the two body decay width $\Gamma_{2}$ of the inflaton to SM particles dominates its total width $\Gamma_{\text {tot }}$. (As can be seen from (2) generically a two body decay (into scalar) gives the dominant contribution to the total decay width.) As discussed in section [3] we further estimate all relevant SM processes by the decay of the inflaton to scalars $\psi$. For this we assume the simple model

$$
\mathcal{L}=\frac{1}{2} \partial_{\mu} \phi \partial^{\mu} \phi-\frac{m_{\phi}^{2}}{2} \phi^{2}+\frac{1}{2} \partial_{\mu} \psi \partial^{\mu} \psi-\frac{m_{\psi}^{2}}{2} \psi^{2}+\frac{\lambda}{2} \phi \psi{ }^{2} .
$$

Moreover, in the physical situations of interest in the main text $m_{\phi} \gg m_{\psi}$, hence we consider the massless limit for $\psi$. The width of the two body decay $\phi \rightarrow \psi \psi$ is then given by

$$
\Gamma_{2}=\frac{\lambda^{2}}{32 \pi m_{\phi}} .
$$

The relevant diagrams for the three body decays involving a KK mode are given in Figure 1, and we define $\Gamma_{3, i}$ as

$$
\Gamma_{3, i}=\int \frac{d\left|M_{i}\right|^{2}}{128 \pi^{3} m_{\phi}} d E_{2} d E_{3}, \quad i=\operatorname{rad}, \text { gr. }
$$

For the estimates of the amplitudes, we need to make a distinction between KK radions and gravitons.

The amplitude for the radion producing reaction $\phi\left(k_{1}\right) \rightarrow \operatorname{rad}\left(k_{2}\right)+\psi\left(k_{3}\right)+\psi\left(k_{4}\right)$ reads (in the same ordering as the diagrams given in Fig. 11)

$$
i M_{\mathrm{rad}}=-i \kappa \omega \lambda\left(\frac{k_{1} \cdot k_{2}+m_{\phi}^{2}}{2 k_{1} \cdot k_{2}-m_{K K}^{2}}-2+\frac{k_{2} \cdot k_{3}}{2 k_{2} \cdot k_{3}+m_{K K}^{2}}+\frac{k_{2} \cdot k_{4}}{2 k_{2} \cdot k_{4}+m_{K K}^{2}}\right),
$$

where we have used $\kappa^{2} M_{\mathrm{p}}^{2}=16 \pi$ and the normalization factor $\omega^{2}=\frac{2}{3(d+2)}$ of the radion 32. By computing the relevant phase space integral and summing over $d$ identical radions, we can finally obtain the full expression for $C_{\text {rad }}(x)$. Since its form is rather complicated, we have plotted this function for $d=3,4$, and 6 in Fig. 3. Its asymptotic expression reads

$$
C_{\mathrm{rad}}(x) \simeq \frac{2 d}{3 \pi(d+2)} \ln \left(\frac{1}{x}\right), \quad \text { for } \quad x=\frac{m_{K K}^{\mathrm{rad}}}{m_{\phi}} \ll 1 .
$$


The matrix element for graviton production $\phi\left(k_{1}\right) \rightarrow \operatorname{gr}\left(k_{2}\right)+\psi\left(k_{3}\right)+\psi\left(k_{4}\right)$ can be obtained in a similar fashion using the diagrams of Fig. [1 However the amplitude is now a tensor quantity. Furthermore, since $m_{\max }^{\mathrm{gr}}$ is significantly smaller than $m_{\max }^{\mathrm{rad}}$ (see (6) ) we may neglect the masses of the KK gravitons. The amplitude for the KK gravitons then becomes

$$
\begin{aligned}
i M_{\mu \nu}^{\mathrm{gr}} & =i \frac{\kappa \lambda}{2}\left(-\frac{m_{\phi}^{2} \eta_{\mu \nu}+C_{\mu \nu \rho \sigma} k_{1}^{\rho}\left(k_{1}^{\sigma}-k_{2}^{\sigma}\right)}{2 k_{1} \cdot k_{2}}+\frac{C_{\mu \nu \rho \sigma}\left(k_{3}^{\rho}+k_{2}^{\rho}\right) k_{3}^{\sigma}}{2 k_{2} \cdot k_{3}}\right. \\
& \left.+\frac{C_{\mu \nu \rho \sigma}\left(k_{4}^{\rho}+k_{2}^{\rho}\right) k_{4}^{\sigma}}{2 k_{2} \cdot k_{4}}+\eta_{\mu \nu}\right), \quad \text { with } \quad C_{\mu \nu \rho \sigma}=\eta_{\mu \rho} \eta_{\nu \sigma}+\eta_{\mu \sigma} \eta_{\nu \rho}-\eta_{\mu \nu} \eta_{\rho \sigma} .
\end{aligned}
$$

This gives for the KK gravitons in the massless approximation

$$
C_{\mathrm{gr}}(x)=\frac{4}{3 \pi} \ln \left(\frac{1}{x}\right) \quad x=\frac{m_{K K}^{\mathrm{rad}}}{m_{\phi}} \ll 1 .
$$

Here we get a $\log \left(m_{\phi} / E\right)$ dependence, where $E$ is the minimum energy carried by the graviton. To avoid an infrared singularity, we assumed that the graviton has some minimum energy, which is taken to be the mass $m_{K K}^{\text {gr }}$.

\section{References}

[1] N. Arkani-Hamed, S. Dimopoulos and G. Dvali, Phys. Lett B 429, 263 (1998) hep-ph/9803315; Phys. Rev. D 59, 086004 (1999) hep-ph/9807344;

I. Antoniadis, N. Arkani-Hamed, S. Dimopoulos and G. Dvali, Phys. Lett. B 436, 257 (1998) hep-ph/9804398.

[2] For some early ideas, see: I. Antoniadis, Phys. Lett. B 246, 377 (1990);

I. Antoniadis, K. Benakli and M. Quirós, Phys. Lett. B 331, 313 (1994) hep-ph/9403290;

K. Benakli, Phys. Rev. D 60, 104002 (1999) hep-ph/9809582; Phys. Lett. B 447, 51 (1999) hep-th/9805181.

[3] For reviews, see: V. A. Rubakov, hep-ph/0104152,

A. Pérez-Lorenzana, hep-ph/0008333

[4] C. D. Hoyle et al, hep-ph/0011014.

[5] G. F. Giudice, R. Rattazzi and J. D. Wells, in Ref. 8].

[6] K. R. Dienes, Phys. Rev. Lett. 88, 011601 (2002) hep-ph/0108115. 
[7] N. Kaloper, J. March-Russell, G. D. Starkman and M. Trodden, Phys. Rev. Lett. 85, 928 (2000) hep-ph/0002001.

[8] T. G. Rizzo, Phys. Rev. D 59, 115010 (1999) hep-ph/9901209;

G. F. Giudice, R. Rattazzi and J. D. Wells, Nucl. Phys. B 544, 3 (1999) hep-ph/9811291;

E. A. Mirabelli, M. Perelstein and M. E. Peskin, Phys. Rev. Lett. 82, 2236 (1999) hep-ph/9811337;

J. L. Hewett, Phys. Rev. Lett. 82, 4765 (1999) hep-ph/9811356;

V. Barger, T. Han, C. Kao and R. J. Zhang, Phys. Lett. B 461, 34 (1999) hep-ph/9905474.

[9] For a review, see: K. A. Olive, G. Steigman and T. P. Walker, Phys. Rept. 333, 389 (2000) astro-ph/9905320.

[10] M. Kawasaki, K. Kohri and N. Sugiyama, Phys. Rev. Lett. 82, 4168 (1999) astro-ph/9811437; Phys. Rev. D 62, 023506 (2000) astro-ph/0002127.

[11] G. Dvali and S. H. H. Tye, Phys. Lett. B 450, 72 (1999) hep-ph/9812483.

[12] N. Kaloper and A. Linde, Phys. Rev D 59, 101303 (1999) hep-th/9811141.

[13] N. Arkani-Hamed, S. Dimopoulos, N. Kaloper and J. March-Russell, Nucl. Phys. B 567, 189 (2000) hep-ph/9903224;

A. Mazumdar, Phys. Lett. B 469, 55 (1999) hep-ph/9902381;

J. Cline, Phys. Rev. D 61, 023513 (2000) hep-ph/9904495.

[14] R. N. Mohapatra, A. Pérez-Lorenzana and C. A. de S. Pires, Phys. Rev. D 62, 105030 (1999) hep-ph/0003089.

[15] M. Kawasaki and T. Moroi, Prog. Theor. Phys. 93, 879 (1995) hep-ph/9403364;

S. Sarkar, Rep. Prog. Phys. 59, 1493 (1996) hep-ph/9602260.

[16] A. Mazumdar and A. Pérez-Lorenzana, Phys. Lett. B 508, 340 (2001) hep-ph/0102174.

[17] K. Benakli and S. Davidson, Phys. Rev. D 60, 025004 (1999) hep-ph/9810280.

[18] R. Allahverdi, K. Enqvist. A. Mazumdar and A. Pérez-Lorenzana, Nucl. Phys. B 618, 277 (2001) hep-ph/0108225.

[19] L. J. Hall and D. Smith. Phys. Rev. D 60, 085008 (1999) hep-ph/9904267.

[20] D. A. Knitten, et al, Astron. Astrophys. Suppl. 120, 615 (1996).

[21] S. C. Kappadath, et al, Astron. Astrophys. Suppl. 120, 619 (1996).

[22] E. W. Kolb and M. S. Turner, The early Universe, New York, Addison-Wesley (1990). 
[23] S. Davidson and S. Sarkar, J. High Energy Phys. 0011, 012 (2000) hep-ph/0009078.

[24] R. Allahverdi and M. Drees, Phys. Rev. D 66, 063513 (2002) hep-ph/0205246.

[25] S. Hannestad, Phys. Rev. D 64, 023515 (2001) hep-ph/0102290.

[26] G. G. Raffelt and S. Hannestad, Phys. Rev. Lett. 87, 051301 (2001) hep-ph/0103201; C. Hanhart, J. A. Pons, D. R. Phillips and S. Reddy, Phys. Lett. B 509, 1 (2001) astro-ph/0102063.

[27] G. G. Raffelt and S. Hannestad, hep-ph/0304029 Phys. Rev. Lett. 88, 071301 (2002) hep-ph/0110067.

[28] R. Allahverdi and M. Drees, Phys. Rev. Lett. 89, 091302 (2002).

[29] J. Ellis, G. B. Gelmini, J. L. Lopez, D. V. Nanopoulos and S. Sarkar, Nucl. Phys. B 373, 399 (1992).

[30] G. Kribs and I. Rotshtein, Phys. Rev. D 55, 4435 (1997) hep-ph/9610468; ibid. D56, 1822 (1997).

[31] R. H. Cyburt, J. Ellis, B. D. Fields and K. A. Olive, astro-ph/0211258.

[32] T. Han, J. Lykken and R. J. Zhang, Phys. Rev. D 59, 105006 (1999) hep-ph/9811350. 